\title{
Non-gravitational motion of the Jupiter-family comet 81P/Wild 2
}

\section{The dynamical evolution}

\author{
M. Królikowska and S. Szutowicz
}

Space Research Centre of the Polish Academy of Sciences, Bartycka 18A, 00-716, Warsaw, Poland

e-mail: mkr@cbk.waw.pl

Received 4 July 2005 / Accepted 29 September 2005

\begin{abstract}
We investigated the influence of the non-gravitational effects on the orbital motion of 81P/Wild 2. First, the non-gravitational accelerations on cometary nucleus throughout all five revolutions around the sun were analysed using both the symmetrical and the asymmetrical $g(r)$-function. Next, the symmetric model was used to examine the past and future dynamical evolution of Wild 2 within the time interval of \pm 8 thousand years. We then introduced a statistical approach. In the most probable scenario for Wild 2 history, a few thousand years ago, this comet was an object with its perihelion close to the Jupiter orbit with an aphelion distance greater than the Neptune orbit: JN class, JE class, JT class, or even larger. Due to the extremely close approach to Jupiter in 1974, its future evolution differs substantially from earlier, so most probably, comet Wild 2 will survive as a typical Jupiter-family comet during the few thousand years that follow. After 8000 yr, however, the chance that the comet Wild 2 will still be a typical JF comet (with $q<3$ AU) drops to 29\%. Similarities between the orbital histories of Wild 2 and comets 16P/Brooks 2 and 67P/Churyumov-Gerasimenko are also discussed.
\end{abstract}

Key words. comets: individual: comet 81P/Wild 2 - comets: general

\section{Introduction}

The Comet 81P/Wild 2 had a close encounter to within $0.0061 \mathrm{AU}$ of Jupiter in September 1974 which perturbed it enough to send it into the inner part of the Solar System. This close approach to about 13 Jovian equatorial radii dramatically affected the orbit enough to change a period of about 40 years down to the period typical of a Jupiter-family comet (6 years) and to reduce the perihelion distance from $4.98 \mathrm{AU}$ to 1.49 AU, as well as the aphelion from 19 AU to 5.2 AU. Owing to reduction of the perihelion distance, the comet was discovered by Paul Wild with the 40/60-cm Schmidt at Zimmerwald on January 6, 1978. Since its discovery, the comet has made only five perihelion passages in June 1978, August 1984, December 1990, May 1997, and September 2003.

The Stardust mission visited 81P/Wild 2 in January 2004 and collected cometary material for return to Earth in 2006. This flyby mission, as well as missions to comets 1P/Halley (Vega \& Giotto 1986), 19P/Borrelly (Deep Space 1, in 2001), and 9P/Tempel 1 (Deep Impact, in 2005) revealed a rather dark surface of a cometary nucleus with limited areas of activity. Among these four comets, however, Wild 2 seems to have the highest percentage of active surface with plenty of small jets (Brownlee et al. 2004) grouped in outgassing areas that cover more than $20 \%$ of the cometary surface (Sekanina et al. 2004). Brownlee et al. (2004) even speculate that "most of the comet surface has been active at one time to another". Images taken by the Stardust spacecraft show the nucleus to be a five-kilometer oblate body, unlike the prolate shapes of the three other comet nuclei. The present nuclei of comets Halley and Borrelly show surface roughness and look more alike than different, although they probably originated from different reservoirs like the Oort cloud (Halley) and the Kuiper belt (Borrelly).

Comet Halley has made many hundreds of passages near the Sun, which has led to the present surface caused by sublimation processes. Comets Borrelly, Tempel 1, and Wild 2 belong to the Jupiter family of comets; but the comet Wild 2 was captured in its current orbit only 30 years ago, and its solar heating history seems to be less intense than for others (Weaver 2004). The nucleus of this comet is rounded, evidence that it is not a collisional fragment of a larger object, and it seems to be much more topologically diverse than other nuclei (Brownlee et al. 2004). Some of its diversities could be explained by the different dynamical evolution. Being recently injected into the inner part of the Solar System, comet 81P/Wild 2 should be relatively new and therefore subjected to only moderate solar heating in its thermal history.

For the paper we carefully examined the non-gravitational (NG) motion of 81P/Wild 2 on the basis of positional observations that covered all five apparitions. The aim of this paper was twofold: first, to derive symmetric and asymmetric NG models of the comet motion (Sect. 3), and second, to use the most 
Table 1. Distribution of the observations of 81P/Wild 2. Numbers of residuals for two recent perihelion passages are decreased by taking normal places of some observations into account. (last column indicated whether normal places were created)

\begin{tabular}{|c|c|c|c|c|c|}
\hline $\begin{array}{l}\text { Perihelion } \\
\text { time }\end{array}$ & Observation interval & $\begin{array}{c}\text { Number } \\
\text { of obs. }\end{array}$ & $\begin{array}{c}\text { Number } \\
\text { of res. }\end{array}$ & $\begin{array}{c}\text { Mean } \\
\text { res. }\end{array}$ & $\begin{array}{l}\text { normal } \\
\text { places }\end{array}$ \\
\hline $1978-6-15$ & $1978-1-06-1979-6-27$ & 177 & 352 & $1{ }^{\prime \prime} 13$ & no \\
\hline $1984-8-20$ & $1983-9-18-1986-9-26$ & 94 & 179 & 1." 43 & no \\
\hline $1990-12-17$ & 1988-9-09-1992-8-02 & 97 & 159 & 0.60 & no \\
\hline \multirow[t]{2}{*}{$1997-5-06$} & $1995-1-02-1998-9-16$ & 642 & 1238 & 0.74 & no \\
\hline & & 234 & 458 & 0.60 & yes \\
\hline \multirow[t]{2}{*}{$2003-9-26$} & 2002-9-05-2004-8-07 & 1252 & 2416 & 0.72 & no \\
\hline & & 228 & 439 & 0.60 & yes \\
\hline Total & 1978-1-06-2004-8-07 & 830 & 1587 & 0.69 & yes \\
\hline
\end{tabular}

adequate and simple NG model of actual motion for Wild 2 in order to examine its past and future NG dynamical evolution within a time interval of \pm 8 thousand years (Sect. 4 ).

\section{Observational material and the method of calculations}

The present investigations are based on the archive positional observations taken from the Minor Planet Center (Cambridge, USA) and from MPECs publicly available on the Web at http://cfa-www . harvard . edu/mpec/RecentMPECs.html. The whole observational material contains 2262 observations covering the time period from January 6, 1978 to August 8, 2004. The observations were selected according to the objective criteria elaborated by Bielicki \& Sitarski (1991) for each of five apparitions separately. The positional observations have a highly nonuniform distribution over the apparitions (Col. 3 in Table 1). This situation leads to weights of individual apparitions that cannot be compared. Thus we created a number of normal places by replacing more than two observations of the same day by one average comet position. This procedure was used for the two last overpopulated apparitions to yield 234 comet positions rather than 642 observations for the perihelion passage in 1997 and 228, rather than 1252, for the return in 2003 (rows 5 and 7 in Table 1). The numbers of residuals obtained by selection and by the normal places procedure are listed in Col. 4 in Table 1. Finally, the total number of 1587 residuals were selected for an orbit improvement, and their quality is represented by the "a priori" rms of 0.69 . The strictly gravitational orbit determined from all five apparitions is fitted with an unacceptably large rms of 8 ".38 compared to the "a priori" rms. This means that NG effects are significant in the orbital motion of 81P/Wild 2.

The equations of cometary motion were integrated numerically using the recurrent power series method (Sitarski 1989, 2002) by taking the perturbations by all the nine planets and by the NG force into account. We linked the observed apparitions of $81 \mathrm{P} /$ Wild 2 and then investigated the dynamical evolution of the comet under the gravitational influence of planets and NG-effects (hereafter called NG evolution). All numerical calculations presented here are based on the Warsaw numerical ephemeris DE405/WAW of the Solar System, consistent with a high accuracy with the JPL ephemeris DE405 (Sitarski 2002).

\section{Models with constant non-gravitational parameters}

\subsection{Standard model}

The standard method (Marsden et al. 1973) was used to estimate the NG force acting on a rotating cometary nucleus due to sublimating ice from its surface. In its original form, the formalism assumes a peak of the outgassing exactly at perihelion. Then three components of the NG acceleration have the form

$F_{i}=A_{i} \cdot g(r), \quad A_{i}=$ const. for $i=1,2,3$,

where the radial component $F_{1}$ is directed along the radius vector, the transverse component $F_{2}$ follows the direction of the orbital motion of the comet, and the normal component $F_{3}$ is perpendicular to the orbital plane. The $g(r)$ function simulates the water ice sublimation rate as a function of the heliocentric distance $r$

$g(r)=\alpha\left(r / r_{\mathrm{o}}\right)^{-m}\left[1+\left(r / r_{\mathrm{o}}\right)^{n}\right]^{-k}$,

where the exponential coefficients $m, n$, and $k$ are equal to $2.15,5.093$, and 4.6142 , respectively. The normalization constant $\alpha=0.1113$ gives $g(1 \mathrm{AU})=1$; the scale distance $r_{0}=2.808 \mathrm{AU}$. From orbital calculations, the model parameters $A_{1}, A_{2}$, and $A_{3}$ can be estimated together with six orbital elements within a given time interval. The model assumes that water sublimates from the whole surface of an isothermal cometary nucleus. The long-exposure images taken by the Stardust mission indicated that the whole surface of comet $81 \mathrm{P} /$ Wild 2 is covered by dozens of regions emitting jets of gas and dust (Brownlee et al. 2004). Thus, a simple model of globally active surface of the comet should be quite adequate for $81 \mathrm{P} /$ Wild 2.

The standard model was used to calculate the NG parameters in short intervals of time covering three consecutive returns of the comet to the Sun. Table 2 shows moderate NG-effects derived from positional data of comet 81P/Wild 2 with somewhat systematic trends in time; here, especially $A_{2}$ seems to decrease during 1978-2004. However, the orbital linkage of all comet apparitions based on the constant parameters $A_{1}, A_{2}, A_{3}$ gives a satisfactory residual of 1 ". 27 (see Model Ia in Table 4). Therefore, we used this model to demonstrate the dynamical evolution of $81 \mathrm{P} /$ Wild 2 within the time interval $\pm 8000 \mathrm{yr}$ (see Sect. 4). The orbital calculations presented in the Tables 2 and 4 are based on the astrometric data reduced accordingly by normal places of observations as shown in Table 1 . The normal component $F_{3}$ is commonly neglected in the orbital computations by many authors. However, one can see that the absolute value of the normal component of NG acceleration, $A_{3}$, is significantly higher than the transverse component, $A_{2}$, for all three time intervals given in Table 2; it is also substantial for a time interval covering all observed apparitions (Model Ia in Table 4). Table 3 compares the parameters $A_{1}$, 
Table 2. NG parameters for the $81 \mathrm{P} /$ Wild 2 determined by linking three consecutive apparitions using symmetric and asymmetric models. Parameters $A_{1}, A_{2}, A_{3}$ are given in units of $10^{-8} \mathrm{AU} \mathrm{day}{ }^{-2}$. The residuals in parentheses represent a strictly gravitational model based on the same set of observations

\begin{tabular}{|c|c|c|c|}
\hline Appearances & 1978-1984-1990 & 1984-1990-1997 & 1990-1997-2003 \\
\hline \multicolumn{4}{|c|}{ Symmetric NG model } \\
\hline & Model NGa & Model NGb & Model NGc \\
\hline$A_{1}$ & $0.09117 \pm 0.01355$ & $0.12411 \pm 0.00975$ & $0.11706 \pm 0.00623$ \\
\hline$A_{2}$ & $0.02290 \pm 0.00051$ & $0.01293 \pm 0.00043$ & $0.00860 \pm 0.00020$ \\
\hline$A_{3}$ & $-0.03942 \pm 0.00778$ & $-0.08410 \pm 0.00858$ & $-0.05171 \pm 0.00588$ \\
\hline $\mathrm{rms}$ & 1.'19 (2.. 45) & $1{ }^{\prime \prime} 03\left(1{ }^{\prime \prime} 55\right)$ & $0.73\left(11^{\prime \prime} 40\right)$ \\
\hline \multicolumn{4}{|c|}{ Asymmetric NG model } \\
\hline$A_{1}$ & $0.09097 \pm 0.01383$ & $0.05918 \pm 0.00630$ & $0.11123 \pm 0.00618$ \\
\hline$A_{2}$ & $0.02352 \pm 0.00384$ & $0.03109 \pm 0.00130$ & $0.03333 \pm 0.00134$ \\
\hline$A_{3}$ & $-0.04001 \pm 0.00797$ & $-0.09942 \pm 0.00686$ & $-0.07536 \pm 0.00578$ \\
\hline$\tau$ (days) & $-2.6 \pm 15.6$ & $-117.3 \pm 9.0$ & $-77.6 \pm 5.3$ \\
\hline $\mathrm{rms}$ & 1.” 19 & $0 ! 91$ & $0 .{ }^{\prime} 66$ \\
\hline umber of & 690 & 796 & 1056 \\
\hline
\end{tabular}

$A_{2}$ extracted from the literature with our results obtained by assuming $A_{3}=0$. Models with $A_{3}=0$ fit the observations with the rms larger of about $0{ }^{\prime} 03$ than respective models with three NG parameters. Table 3 also illustrates how the number of observations (for the fixed arc of observations) and selection procedure affect the derived values of NG parameters. One can notice that the normal place procedure influences the resulting parameters in a moderate way (compare model pairs: A1-A2, B2-B3, C1-C2, and D1-D2).

\subsection{Extended standard model}

A modification of the standard model postulated by Yeomans \& Chodas (1989) allowed the comet's outgassing to peak before or after perihelion. They generalized Eqs. (1) and (2) of the NG effects to an asymmetric case with respect to perihelion by substituting the $g\left(r^{\prime}\right)$ instead of $g(r)$, where $r^{\prime}=$ $r(t-\tau)$. The parameter $\tau$ represents a time shift (measured in days) of the maximum of function $g(r)$ with respect to the perihelion. To obtain the best asymmetric solutions for an individual comet Yeomans \& Chodas (1989) varied the value of $\tau$ by trial and error. They concluded that the asymmetric model of NG acceleration improved orbital solutions in comparison to symmetric models. A method of determining the strict value of $\tau$ as an additional parameter, along with three standard NG parameters $A_{1}, A_{2}$, and $A_{3}$ was first proposed by Sitarski (1994a) and then implemented by him for many short-period comets (among others papers: Sitarski 1994b, 1996). Recently, Chesley \& Yeomans (2004) also developed the four-parameter NG model (with constant $A_{1}, A_{2}$, $A_{3}$, and $\tau$ ) as extended standard model (ESM). Their ESM solution for comet Wild 2 was based on observations spanning the interval between Sept 09, 1988 and Dec. 30, 2003 and led to a pre-perihelion shift of the maximal outgassing by 23 days. Our orbital linkage of these three apparitions is based on the
Table 3. NG parameters $A_{1}$ and $A_{2}$ of $81 \mathrm{P} /$ Wild 2 derived with an assumption that normal component $A_{3}$ is equal to zero. The results derived from the normal places of the observations are distinguished by "with NP". The solutions mark as Model B1 Model C3 and Model C5 are taken from: ssd.jpl.nasa.gov/cgi-bin/da_shm?rec $=900120$ (JPL I), ssd.jpl.nasa.gov/cgi-bin/da_shm?rec=900119 (JPL II), ww.oaa.gr.jp/ oaacs/nk/nk716 (NK 716; Nakano Notes), respectively.

\begin{tabular}{|c|c|c|c|}
\hline $\begin{array}{c}\text { Arc of observations } \\
\text { No of apparitions } \\
\text { rms }\end{array}$ & $\begin{array}{c}A_{1} \\
A_{2} \\
{\left[\times 10^{+8} \mathrm{AU} / \mathrm{day}^{2}\right]} \\
\end{array}$ & Model & References \\
\hline $\begin{array}{c}\text { 1978-01-06-2004-08-08 } \\
5 \text { app (2262 obs.) } \\
\text { rms }=1 \text { I. } 13^{-13}\end{array}$ & $\begin{array}{l}+0.18907 \pm 0.00365 \\
+0.01334 \pm 0.00007\end{array}$ & A1 & $\begin{array}{l}\text { present calc. } \\
\text { without NP }\end{array}$ \\
\hline $\begin{array}{c}\text { 1978-01-06-2004-08-08 } \\
5 \text { app (830 obs.) } \\
\text { rms }=1.30\end{array}$ & $\begin{array}{l}+0.13726 \pm 0.00611 \\
+0.01436 \pm 0.00012\end{array}$ & A2 & $\begin{array}{l}\text { present calc. } \\
\text { with NP }\end{array}$ \\
\hline $\begin{array}{c}\text { 1988-09-09-2004-08-08 } \\
3 \text { app; (1890 obs.) }\end{array}$ & $\begin{array}{l}+0.1055 \\
+0.0327\end{array}$ & B1 & JPL I \\
\hline $\begin{array}{c}\text { 1988-09-09-2004-08-08 } \\
3 \text { app (1991 obs.) } \\
\text { rms }=0 .^{\prime} 81\end{array}$ & $\begin{array}{l}+0.12637 \pm 0.00485 \\
+0.00782 \pm 0.00015)\end{array}$ & B2 & $\begin{array}{l}\text { present calc. } \\
\text { without NP }\end{array}$ \\
\hline $\begin{array}{c}\text { 1988-09-09-2004-08-08 } \\
3 \text { app (559 obs.) } \\
\text { rms }=0 .^{\prime} 76 \\
\end{array}$ & $\begin{array}{l}+0.11876 \pm 0.00645 \\
+0.00859 \pm 0.00021\end{array}$ & B3 & $\begin{array}{l}\text { present calc. } \\
\text { with NP }\end{array}$ \\
\hline $\begin{array}{c}\text { 1978-01-06-1998-09-16 } \\
4 \text { app (1010 obs.) } \\
\text { rms }=1 . .08\end{array}$ & $\begin{array}{l}+0.14157 \pm 0.00544 \\
+0.01748 \pm 0.00019\end{array}$ & $\mathrm{C} 1$ & $\begin{array}{l}\text { present calc. } \\
\text { without NP }\end{array}$ \\
\hline $\begin{array}{c}\text { 1978-01-06-1998-09-16 } \\
4 \text { app (602 obs.) } \\
\text { rms }=11^{\prime \prime} 20\end{array}$ & $\begin{array}{l}+0.13789 \pm 0.00721 \\
+0.01717 \pm 0.00023\end{array}$ & $\mathrm{C} 2$ & $\begin{array}{l}\text { present calc. } \\
\text { with NP }\end{array}$ \\
\hline $\begin{array}{c}\text { 1978-01-06-1998-09-16 } \\
4 \text { app; (857 obs.) }\end{array}$ & $\begin{array}{l}+0.1217 \\
+0.0187\end{array}$ & $\mathrm{C} 3$ & JPL II \\
\hline $\begin{array}{c}\text { 1978-1998 (798 obs.) } \\
4 \text { app; rms }=0 .^{\prime} 8\end{array}$ & $\begin{array}{c}+0.11 \\
+0.0193\end{array}$ & $\mathrm{C} 4$ & MPC 40671 \\
\hline $\begin{array}{c}1978-1998 \text { (847 obs.) } \\
4 \text { app; rms }=0 .^{\prime} 85\end{array}$ & $\begin{array}{c}+0.112 \pm 0.007 \\
+0.01919 \pm 0.00019\end{array}$ & $\mathrm{C} 5$ & NK 716 \\
\hline $\begin{array}{c}\text { 1995-01-02-2004-08-08 } \\
2 \text { app (1894 obs.) } \\
\text { rms }=0 .{ }^{\prime} 77\end{array}$ & $\begin{array}{l}+0.13100 \pm 0.00727 \\
+0.03347 \pm 0.00350\end{array}$ & D1 & $\begin{array}{l}\text { present calc. } \\
\text { without NP }\end{array}$ \\
\hline $\begin{array}{c}\text { 1995-01-02-2004-08-08 } \\
2 \text { app (462 obs.) } \\
\text { rms }=1 . ' 66\end{array}$ & $\begin{array}{l}+0.13537 \pm 0.01702 \\
+0.03378 \pm 0.00479\end{array}$ & D2 & $\begin{array}{l}\text { present calc. } \\
\text { with NP }\end{array}$ \\
\hline $\begin{array}{c}\text { 1995-01-02-2004-02-27 } \\
2 \text { app (1694 obs.) } \\
\text { rms }=0 . ' 48\end{array}$ & $\begin{array}{l}+0.04440 \pm 0.00771 \\
-0.01367 \pm 0.00367\end{array}$ & D3 & $\begin{array}{l}\text { Cometary Notes } \\
\text { of Bureau des longi- } \\
\text { tudes (note no 008) }\end{array}$ \\
\hline
\end{tabular}

observations until Aug. 7, 2004 and resulted in the outgassing peak 77 days before perihelion (see Table 2).

The orbital period of comet Wild 2 systematic increases due to NG perturbations. The systematic trend in NG parameters, especially $A_{2}$, was also pointed out by Sekanina (2003). According to orbital solutions given in Table 2, the perihelion delay, $\Delta P$, decreased from 0.021 to 0.007 days within the 
Table 4. NG parameters and orbital elements for the 81P/Wild 2 derived from all positional observations (five apparitions). The parameters $A_{1}, A_{2}, A_{3}$ are given in units of $10^{-8} \mathrm{AU} \mathrm{day}^{-2}$. Angular elements $\omega, \Omega, i$ refer to Equinox J2000.0 (Epoch: 20050130). Numbers in parentheses denote uncertainties: $1.59101828(40) \equiv 1.59101828 \pm$ 0.00000040 . Model Ia represents a nominal NG orbit, which is used as a starting orbit for NG dynamical integration

\begin{tabular}{ccc}
\hline \hline & Model Ia & Model Ib \\
\hline$A_{1}$ & $0.13625 \pm 0.00596$ & $0.13883 \pm 0.006238$ \\
$A_{2}$ & $0.014370 \pm 0.000115$ & $0.028179 \pm 0.000966$ \\
$A_{3}$ & $-0.032473 \pm 0.003682$ & $-0.049789 \pm 0.004205$ \\
$\tau$ (days) & - & $-38.3 \pm 2.9$ \\
$T$ & $20030925.95455(30)$ & $20030925.95370(30)$ \\
$q$ & $1.59101828(40)$ & $1.59102300(49)$ \\
$e$ & $0.53867896(9)$ & $0.53867748(13)$ \\
$\omega$ & $41^{\circ} 78618(33)$ & $41^{\circ} 78581(28)$ \\
$\Omega$ & $136^{\circ} 13943(32)$ & $136^{\circ} 13963(27)$ \\
$i$ & $3^{\circ} 24003(2)$ & $3^{\circ} 23995(2)$ \\
$\mathrm{rms}$ & $1^{\prime \prime} .27$ & $1^{\prime \prime} 21$ \\
\hline
\end{tabular}

observational intervals 1978-1990 and 1990-2003, respectively. However, the value of $A_{2}$ depends on both the investigated time interval and the applied model. The asymmetric models resulted in a peak of the $g$-like function before perihelion and larger values of parameter $A_{2}$ compared to the standard models.

The solution of our ESM (Model Ib) for all apparitions decreases the rms in comparison to a symmetric model and qualitatively agrees with the observed perihelion asymmetry in comet Wild 2 brightness. Sekanina (2003) discusses the composite light curve of the comet from 1978-1997 and showed that it is not symmetrical relative to perihelion. The comet reaches maximum brightness about three weeks before perihelion, and the pre-perihelion branch of the light curve grows steeper as its post-perihelion branch decreases. Sekanina gives an average rate of 1.8 mag per 100 days for preperihelion brightening and $1.1 \mathrm{mag}$ per 100 days for postperihelion fading. In our approach, the comet asymmetry in its outgassing activity with respect to perihelion is modelled by introducing a time shift $\tau$ for the $g(r)$ function.

The modelled NG perturbation fitted into all positional observations gives a sublimation maximum about 38 days before perihelion (Model Ib in Table 4), and even 86 days for the positional data covering four apparitions 1978-1997, which is substantially greater than the maximum of the cumulative light curve shift discussed by Sekanina (2003). However, Farnham $\&$ Schleicher (2005) estimate that the production rate peaks 11-12 weeks before perihelion. One should notice that the pre- and post-maximum branches of the shifted $g(r)$ function are symmetrical to each other in contrast to the asymmetric slopes of Wild 2's brightness curve. Thus the modelled shift of the outgassing maximum can differ from the observed one. The effect will be discussed in detail in the second paper of this series. Our NG models indicate that the time-shift of the
Table 5. Starting gravitational orbits for the dynamical evolution presented in Fig. 1. Angular elements $\omega, \Omega, i$ refer to Equinox J2000.0 (Epoch: 20050130).

\begin{tabular}{clll}
\hline \hline Model & \multicolumn{3}{c}{ Orbital elements } \\
& & \\
\hline GRb & $T=20030925.94916$ & $q=1.59103279$ & $e=0.53867255$ \\
(future & $\omega=41^{\circ} 78353$ & $\Omega=136^{\circ} 14150$ & $i=3^{\circ} 24002$ \\
evolution) & arc: $1988-2004$ (3 app) & number of obs.: 559 & res $=1^{\prime \prime} 40$ \\
& & & \\
GRb & $T=20030925.91486$ & $q=1.591012972$ & $e=0.53867072$ \\
(past & $\omega=41^{\circ} 78901$ & $\Omega=136^{\circ} 13960$ & $i=3^{\circ} 24037$ \\
evolution) & arc: $1978-1992(3$ app) & number of obs.: 368 & res $=2^{\prime \prime} 45$ \\
\hline
\end{tabular}

maximum outgassing depends on the observational interval taken into account.

\section{Dynamical evolution of Wild 2}

\subsection{Evolution of the nominal orbit}

The basic dynamical evolution of comet 81P/Wild 2 was performed for the NG orbit (Model Ia) and was followed up to 8 thousand years from the starting epoch of integration (Jan. 30, 2005). Due to the sublimation and disintegration of a cometary body, the lifetimes of a typical comet is limited to only $\sim 10$ thousand years. Thus, it seems reasonable to consider not much longer time intervals of the orbital motion of Wild 2 than several thousand yr proposed. This orbital evolution (hereafter, nominal NG orbit evolution) was compared with the evolutionary track obtained for a strictly gravitational evolution starting from Model GRb; in Table 5, the strictly gravitational orbit based on all 5 apparitions gives an unacceptably large residual of 8 ". 38 and poorly represents the actual motion of Wild 2. In the following, the past cometary evolution will be discussed in terms of backwards integration of the osculating orbit.

Evolutionary tracks of the nominal NG orbit attributed to Model Ia, as well as the strictly gravitational orbit represented by Models GRb, are shown by thick curves in Fig. 1. Prior to 1974 , the comet moved between a perihelion at $\sim 5.0 \mathrm{AU}$ and an aphelion at $\sim 19 \mathrm{AU}$ on the orbit inclined $19^{\circ}$ to ecliptic (Models Ia, GRb). These values differ slightly from the result given by Sekanina \& Yeomans (1985), for the comet orbited between a perihelion at 4.9 AU and an aphelion at $25 \mathrm{AU}$. Their calculations, however, were based on the starting orbit determined from two apparitions in 1978 and 1984. One can see that further evolutions of the two starting orbits (Models Ia and GRb) are different. Before 1974, the earlier orbit of Model Ia had been moderately modified for at least eight thousands years around values of $\sim(4.5-5)$ AU for perihelion distance, $0.5-0.6$ for eccentricity, and 13-20 deg for orbit inclination, which gives a semimajor axis of about 8-11 AU and aphelion distance between 14-19 AU. The lower limits of perihelion distance and eccentricity (also semimajor axis and aphelion distance) derived from Model GRb are below those for Model Ia (see thin solid lines in Fig. 1). 


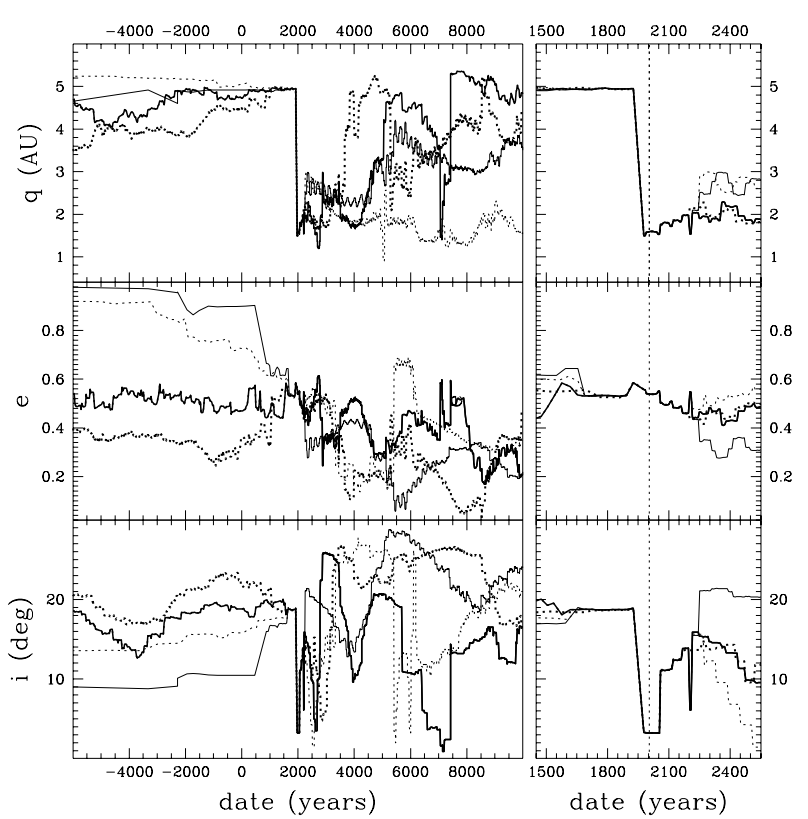

Fig. 1. Comparison between the dynamical evolution of the orbital elements $q, e$, and $i$ of 81P/Wild 2 for the nominal NG orbit of Model Ia (thick solid lines) and the strictly gravitational orbit of Model GRb (thick dotted lines), as well as the typical individual history for two of the 100 clones with a nominal NG orbit. For starting the NG orbit, the NG perturbations were included in the dynamical evolution. The evolution was performed backwards and forwards up to $8000 \mathrm{yr}$ from the starting moment of integrations (Jan. 30, 2005). The evolution of clone \#26 is shown by thin solid curve.

The very close encounter with Jupiter in 1974 dramatically changed the comet's trajectory, as the orbit shrank dramatically to an orbit that is typical of short-period comets belonging to Jupiter family. Its perihelion distance dropped to $1.49 \mathrm{AU}$, the aphelion reduced to 5.24 AU from the Sun, and orbital inclination decreased to $3^{\circ}$. Future orbital evolution tracks also ran differently. For example, the evolution of Model Ia exhibits two separate discrete periods of time with perihelion distance below 3 AU between years: 1974-4600 and 7060-7424, whereas the perihelion distance of Model GRb evolution stays below 3.0 AU within time intervals 1974-3660 and 5280-6050.

Figure 2 shows in detail how large planets control the evolution of $81 \mathrm{P} /$ Wild 2 . The classification scheme proposed by Horner et al. (2003) was applied. Let us discuss the NG evolution of nominal orbit of Model Ia presented on the left-hand side of this figure. Before 1974 the cometary orbits were placed in the JU region in the upper panel of Fig. 2. Thus, during the evolution prior to the comet's discovery, the perihelion was controlled by Jupiter, and the aphelion was placed in the Uranus zone of control. That the perihelion distance was under the control of Jupiter kept its Tisserand parameter $T_{\mathrm{J}}$ below the boundary value of 2.8 that divides loosely bound Jupiterfamily comets $(2.5<q<2.8$; class III in Horner et al. 2003) from tightly bound JFC ( $q>2.8$; class IV therein). Strictly speaking, Horner et al. reserved the term "comet" only for objects moved on orbits with perihelion distance $q<4.0$ AU. Indeed just before its discovery, the comet Wild 2 had an
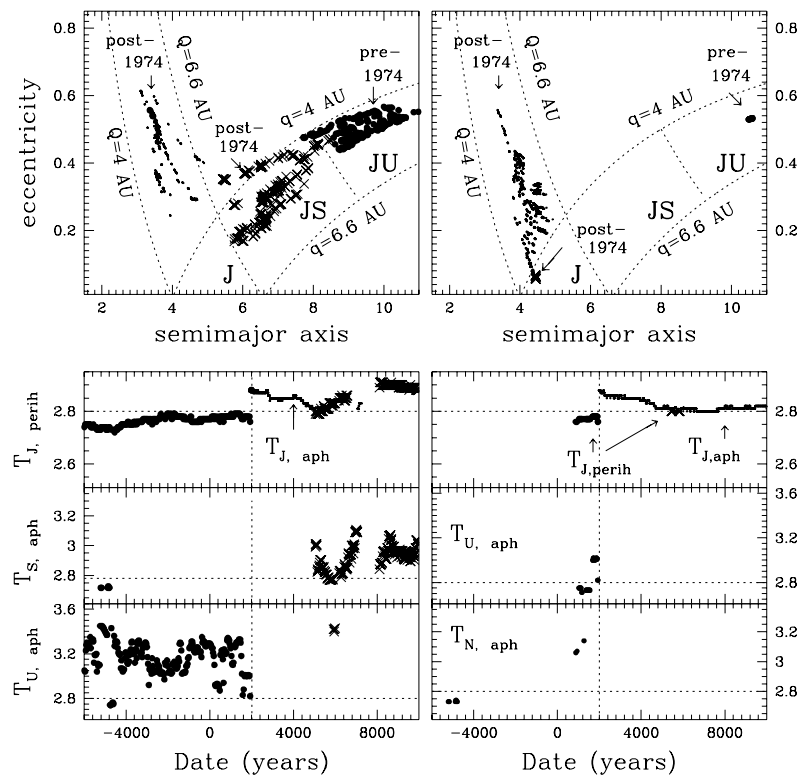

Fig. 2. Upper panel: plot of eccentricity versus semimajor axis for the NG evolution of comet 81P/Wild 2 corresponding to the nominal orbit of Model Ia (the left-hand side) and one of randomly selected orbits of Model Ia (clone \#26) (the right-hand side). The dashed thin curves mark the boundaries of the aphelion or perihelion zones controlled by Jupiter. Jupiter's zone of control is taken as three times the Hill radii. In the zone of $4 \mathrm{AU}<q<6.6 \mathrm{AU}$ to the right of line $Q>4 \mathrm{AU}$, the regions belonging to SP comet's categories are: J (objects for which both perihelion and aphelion are under Jupiter's control), JS (perihelion is under Jupiter's and the aphelion under Saturn's control), and JU (perihelion - as previously, and the aphelion under Uranus's control) (Horner et al. 2003). The evolution was performed backwards and forwards up to $8 \mathrm{kyr}$. Lower panel: changes of Tisserand parameter, $T_{\mathrm{J}, \text { perih }}\left(T_{\mathrm{J}, \mathrm{aph}}\right), T_{\mathrm{S} \text {,aph }}, T_{\mathrm{U}, \text { aph }}$ during a time interval in which the perihelion falls in Jupiter's zone of control and/or the aphelion falls in either Jupiter's Saturn's, Uranus', or Neptune's zone of control. The horizontal dashed lines show the boundaries that differentiate between SP comets using the Tisserand parameter. After Horner et al. (2003), two of this four-fold division are denoted by III (third class having $2.5 \mathrm{AU} \geq T_{\mathrm{J}}>2.8 \mathrm{AU}$ ) and IV (fourth class having $T_{\mathrm{J}} \geq 2.8 \mathrm{AU}$ ) correspond to loosely bound Jupiter-family comets and tightly bound Jupiter-family comets, respectively.

orbit that was similar to the orbits of some Centaurs objects; for example, 2000 EC98 moves in an orbit with a $35 \mathrm{yr}$ period that is characterized by $q \simeq 5.8 \mathrm{AU}, e \simeq 0.46$, and $i \simeq 4^{\circ}$ (aphelion: $Q \simeq 16 \mathrm{AU}$ ). However, in the current analysis, we used the term comet for Wild 2, keeping in mind its present-day orbital behavior. After 1974, the orbit - typical of Jupiter-family comets - remains almost $3000 \mathrm{yr}$ within the zone 4.0 $\mathrm{AU}<Q<6.6 \mathrm{AU}$, which is placed on the left and above the $J$ region in the upper-left panel of Fig. 2. After $\sim 4600 \mathrm{AD}$ it evolves to the right of this figure, to JS region, where usually stays until the end of its future evolution; only one transient return was noticed for $\sim 500 \mathrm{yr}$ to the left zone, where aphelion is controlled by Jupiter. 
Table 6. Mean values and dispersions of NG parameters and of orbital elements derived for 100 randomly selected orbits of 81P/Wild 2 (Epoch: 20050130; Equinox: J2000.0).

\begin{tabular}{rrrrrr}
\hline \hline$\left\langle A_{1}\right\rangle$ & $\left\langle A_{2}\right\rangle$ & $\left\langle A_{3}\right\rangle$ & & & \\
\hline 0.13670 & 0.014373 & -0.03219 & & & \\
+0.01346 & +0.000326 & +0.01299 & & & \\
-0.02706 & -0.000222 & -0.01005 & & & \\
\hline$\langle T\rangle$ & $\langle q\rangle$ & $\langle e\rangle$ & $\langle\omega\rangle$ & $\langle\Omega\rangle$ & $\langle i\rangle$ \\
20030925.95456 & 1.59101830 & 0.53867895 & 41.78614 & 136.13947 & 3.24003 \\
+0.00126 & +0.00000128 & +0.00000022 & +0.00061 & +0.00113 & +0.00006 \\
-0.00065 & -0.00000098 & -0.00000031 & -0.00115 & -0.00052 & -0.00005 \\
\hline
\end{tabular}

\subsection{Evolution of the cloned orbits}

To better understand the past and future evolution of Wild 2, a statistical approach was introduced. The sample of 100 clones of the nominal NG orbit (Model Ia) of the comet was constructed according to the method described by Sitarski (1998). Each individual orbital clone fits the observations with the rms no larger than rms for a nominal orbit. Thus each of them could be an actual orbit of Wild 2. The derived range of orbital elements and of constant NG parameters $A_{1}, A_{2}, A_{3}$ are given in Table 6. Next, each orbital clone was integrated backwards and forwards up to 8 thousand years (like the nominal orbit was). The differences in the orbital NG evolution between some clones are clearly visible in Fig. 1 where the history of the nominal orbit is drawn by a thick curve. The evolutionary tracks of chosen clones represent the most typical history of 100 randomly selected orbits. One can see that the orbital evolution of $81 \mathrm{P}$ is well-defined in the period of $[-300 ;+250]$ years (right-hand side of Fig. 1), while outside this time-interval dynamical evolution starts to be chaotic. For longer time intervals, it is possible, however, to speculate about the most probable history of the comet. It has turned out that the sample of 100 clones gives well-defined statistics for the past and future evolution of orbital elements (Fig. 5).

Close encounters with Jupiter exhibit a non-symmetric time distribution with respect to the present day. To consider the cumulative numbers of very close approaches to Jupiter, the event of 1974 - present in all the past evolutions of 100 clones - should not be taken into account. Then, the very close encounters with Jupiter to within 0.05 AU/0.1 AU are significantly more frequent (78 events/228 events) in the future evolution than they were in the past (27 events/66 events; Fig. 3 ). Similarly, the future encounters to within $0.4 \mathrm{AU}$ (or $0.85 \mathrm{AU}$ ) are significantly more frequent than the past approaches to that planet (panels 1-3 in Fig. 3). This is at least partly a consequence of the comet's shorter orbital period after 1974. Similar analysis for Saturn gives quite a symmetric distribution of close encounters to within $0.85 \mathrm{AU}$ for forwards (128 events) and backwards (129 events) integrations (Fig. 4).

The changes in orbital element distributions throughout the backwards and forwards evolutions are presented in Fig. 5. Within a time interval of $\mp 8000$ years, dynamical evolution of

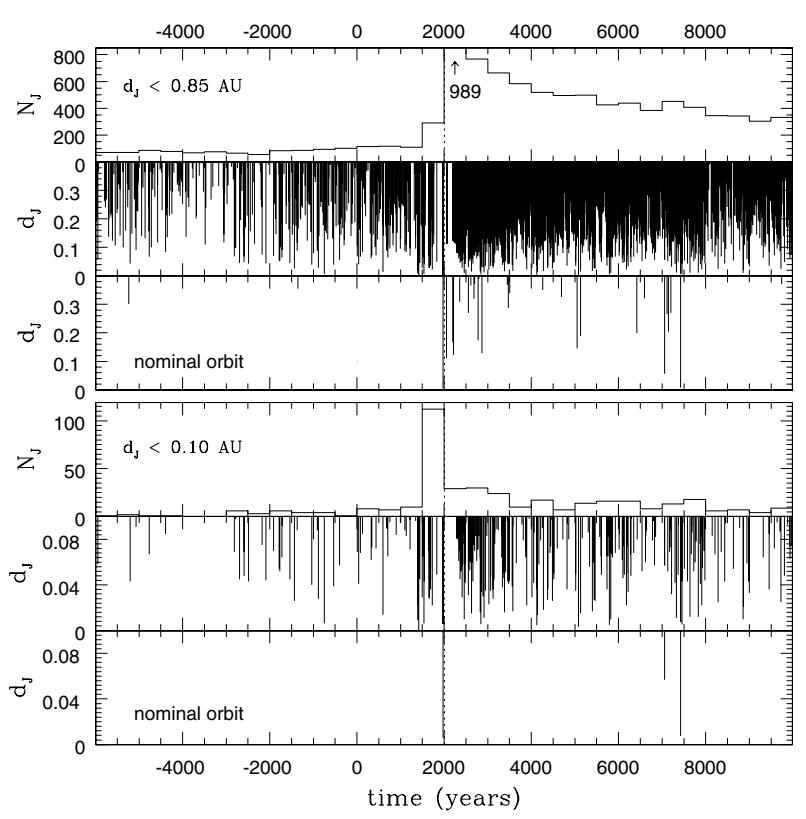

Fig. 3. Cumulative distribution of all close approaches of the comet to Jupiter that occurred during the evolution of 100 clones of NG orbit constructed from Model Ia (panels 1 and 4 from the top). The $y$-axis on the second and fifth panels (marks by $d_{\mathrm{J}}$ ) shows the depths of individual close encounters of the comet with Jupiter; close encounters that occurred during the evolution of a nominal orbit are drawn separately on the third and sixth panels. The evolution was performed backwards and forwards up to $8000 \mathrm{yr}$. The starting moment of integrations is shown by a dotted vertical line.

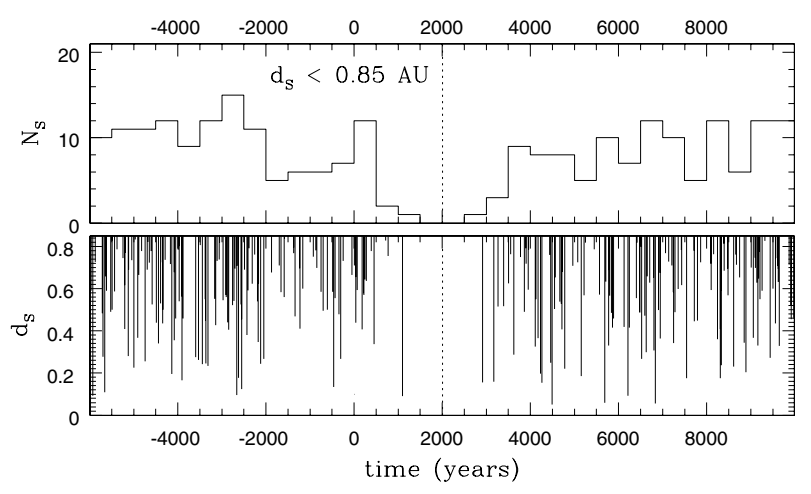

Fig. 4. The same as Fig. 3 for close encounters with Saturn.

sets of 100 randomly selected orbits could be summarized as follows.

1. Due to an extremely close approach to Jupiter in 1974, the past evolution differs substantially from the future evolution.

2. Including the nominal orbit itself, $74 \%$ of the clones evolved backwards always with perihelion distances greater than $4 \mathrm{AU}$. Among the remaining 26\% are evolutions with longer or shorter time intervals where $q$ reaches $3 \mathrm{AU}(13 \%), 2 \mathrm{AU}(4 \%)$ and even $q<2 \mathrm{AU}(9 \%)$.

3. After $8000 \mathrm{yr}, 53 \%$ of the clones evolved backwards to orbits with eccentricities that are higher than 0.7 , and aphelion distances are close to the orbit of Neptune or even farther from the Sun (Fig. 5). Thus in the most probable 
scenario of the clones history, a few thousand years ago, comet Wild 2 was an object of JN class $(22.5 \mathrm{AU}<Q<$ 33.5 AU; Horner et al. nomenclature) with eccentricities $0.64<e<0.74$ (8\%), JE (33.5 AU $<Q<60 \mathrm{AU})$ with $0.74<e<0.85(14 \%)$, or JT $(Q>60 \mathrm{AU})$ with $0.85<e<$ $0.90(11 \%)$, where E stands for the Edgeworth-Kuiper belt region and $\mathrm{T}$ for the trans-Neptunian region. The next 20\% of the clones evolved to even more eccentric orbits $(e>$ $0.9)$ with the perihelion close to Jupiter orbit, aphelion distance greater than $100 \mathrm{AU}$, and period greater than 350 years; the aphelion distances (periods) of three clones even increased to about $1000 \mathrm{AU}$ (about 10000 years).

4. Among the remaining $47 \%$ of clones, at the end of their past evolution their aphelia group around Jupiter (6\% of clones), Saturn (11\%), and Uranus (30\%; including the case of nominal orbit).

5. Typical future evolution exhibits longer or shorter intervals of small perihelion distances. For example, a nominal orbit evolution has two separate discrete periods of time with a small perihelion distance of $q<3$ AU between years 1974-4600 and 7060-7424, whereas the perihelion distance of clone \#26 is below $3 \mathrm{AU}$ inside about 6 thousand years and for clone \#22 - during complete future evolution (Fig. 1). In these intervals, the aphelia stay in Jupiter's zone of control (Fig. 2, the right-hand side). Thus, with a high probability, the comet Wild 2 will be a typical Jupiter-family comet in the subsequent few thousand years; $64 \%$ of clones stay with $q<3 \mathrm{AU}$ after $2000 \mathrm{yr}$, after $4000 \mathrm{yr}-48 \%$, and after $8000 \mathrm{yr}$ only $29 \%$.

6. Almost $90 \%$ of the clones evolve to orbit with the perihelion and/or aphelion controlled by Jupiter at the end of integration, where $40 \%$ of the clones, including the nominal orbit itself, has a perihelion in the Jupiter zone of control (4.0 AU $<q<6.6 \mathrm{AU}$ ) (Fig. 5, Fig. 1 - nominal orbit). The aphelia fall mainly in the Jupiter (44\% of clones) and Saturn zone of control (27\%; the case of nominal orbit), or Uranus zone (16\%). The remaining $13 \%$ of the aphelia are grouped around Neptune's orbit $(6 \%)$ or farther (7\%). Thus, with the same high probability, comet Wild 2 will stay at an orbit that is typical of JFC or will transform to orbit with perihelion inside Jupiter's zone of control and with an aphelion around Saturn or Uranus (Fig. 5). Among the orbits typical for JFC, about half of the orbits (20\% of all clones) have $q>3 \mathrm{AU}$ at the end of future evolution (for example clone \#37 in Fig. 6).

7. In contrast to the past evolution, there is little prospect for comet Wild 2 to be a long-period comet in the following 8000 years. After 8000 yr, only $10 \%$ of clones evolved to orbits with eccentricities higher than 0.7, and $4 \%$ of clones reached eccentricities greater than 0.85 $(P>200$ years where $q \sim 5 \mathrm{AU}$ ) (middle column in Fig. 5). Examples of such evolutions are shown in Fig. 6 (clones \#64,75), where in both cases orbits evolve to long-period cometary orbits with periods between 1000 and 2000 years.

8. In the future evolution, the perihelion distance stays (typically for a hundred years) inside Earth's orbit for 39 of the 100 clones. In the consequence, the total number

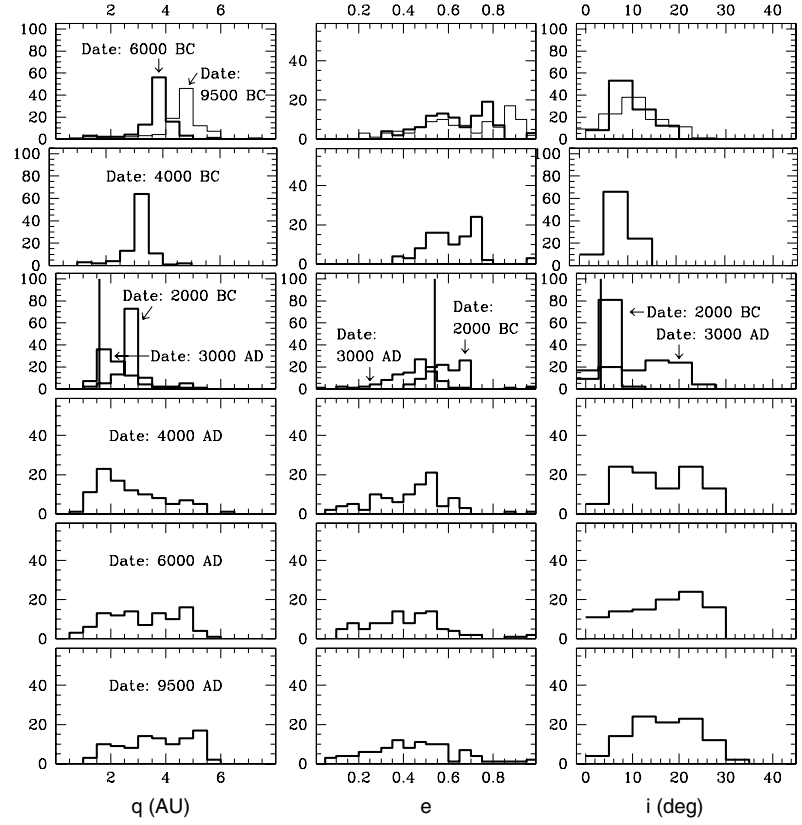

Fig. 5. Evolution of the orbital element distribution for the 100 clones of the nominal orbit corresponding to Model Ia. Each individual row shows the distributions of orbital elements $q$ (left column), $e$ (middle column), and $i$ (right column) of 100 clones for any given moment of evolution, which is specified in the middle panel. The starting distributions of orbital elements are presented in the third row by thick solid line. The thin-dotted distributions in the top panel correspond to $9500 \mathrm{BC}$.
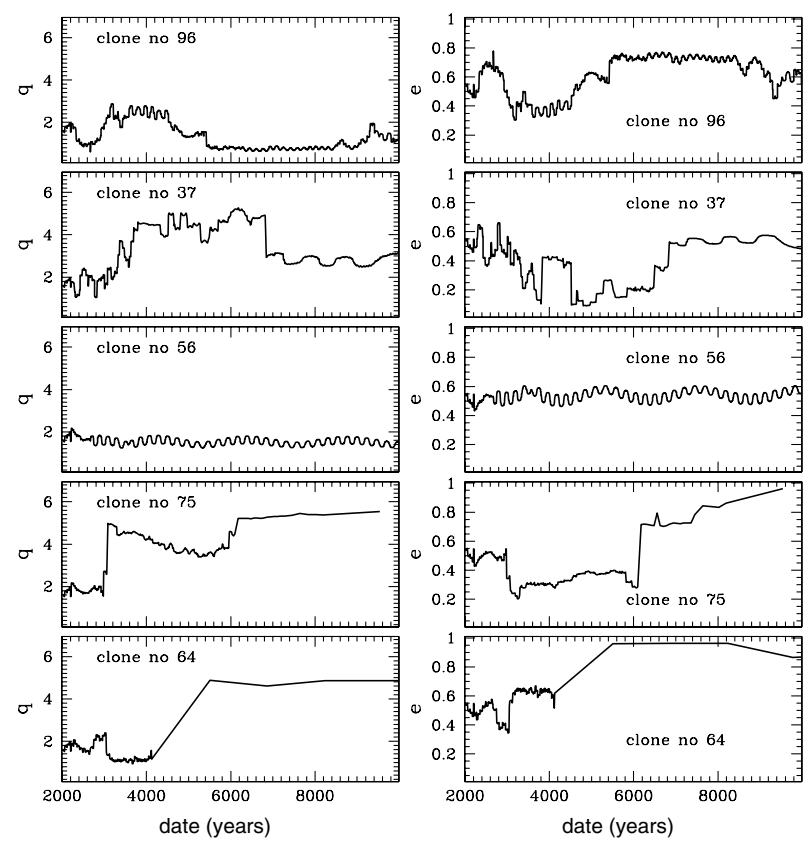

Fig. 6. Individual future non-typical histories of five of the 100 clones.

of 519 close encounters take place with Earth (closer than $0.25 \mathrm{AU}$ ). Two examples of such evolutions are shown in Fig. 6 (clones \#37,96). The first close encounter with Earth occurs in $2330 \mathrm{AD}$ (to within $0.09 \mathrm{AU}$; clone \#37), and the deepest one - in 2441 - to within $0.012 \mathrm{AU}$ (clone \#96, Fig. 6). Similar analysis of past evolutions 


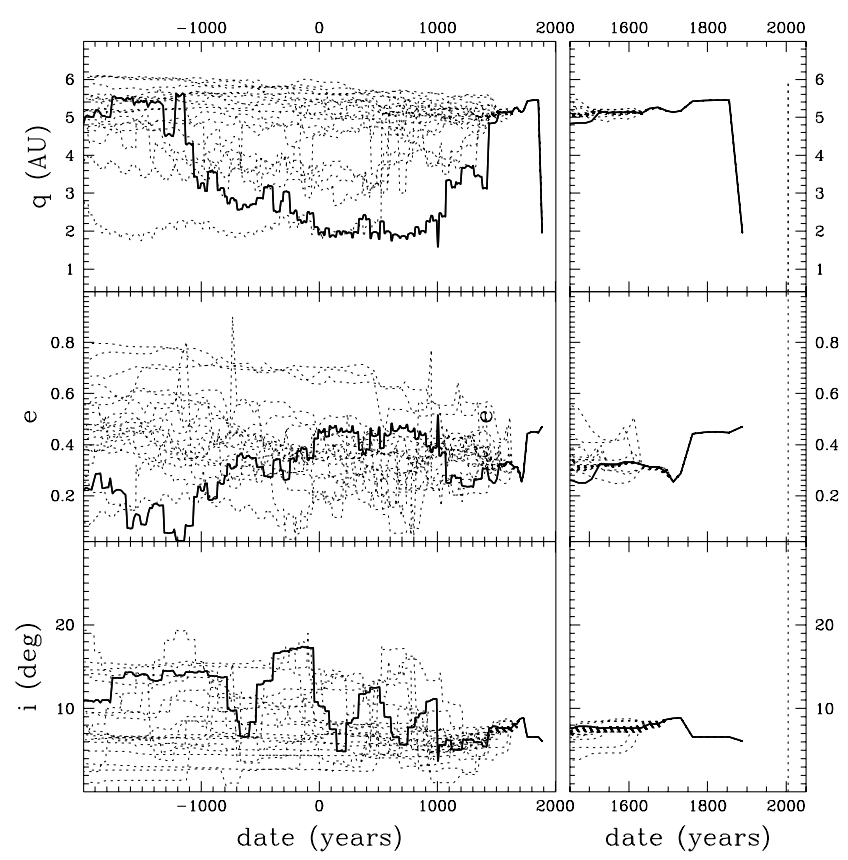

Fig. 7. Past evolution of the orbital elements $q, e$, and $i$ of 20 randomly selected orbits of 16P/Brooks 2 (thin solid lines). The evolution starting from a nominal orbit is shown by thick solid lines. The nominal orbit represents the strictly gravitational solution obtained from the two first apparitions covering the time period 1889-07-06-1896-10-06 (77 observations) and was fitted to the observations with rms of 0 ' 32 .

gives only two clones with close encounters with Earth, where 167 such events occur for one of them (clone \#2) and only 2 for the second (clone \#74). In summary, it seems that comet Wild 2 has a chance of being the Near Earth Comet in the future.

9. Few clones are quickly captured into a resonant orbit with Jupiter for thousands of years - the orbital evolution of clone \#56 is the most spectacular example of such behavior (Fig. 6). This clone is captured into a fairly stable orbit at around a semimajor axis of $3.4 \mathrm{AU}$, which is in the 1:2 resonance with Jupiter. Starting from AD 2600, it remains in this resonance for the whole time of future integration. The short intervals of resonances with Jupiter (the most popular are $1: 2,2: 7,2: 5,5: 7,2: 3,3: 4,1: 1)$ commonly take place in the future evolution of 100 clones. For example, clone \#96 (Fig. 6) exhibits three intervals of different resonances with Jupiter (2400-2700: 1:2 resonance; 3200-4400: 2:3 resonance; 5600-8400 2:5 resonance), while clone \#64 is transferred to a long-period orbit after lasting about $1000 \mathrm{yr}$ in 3:7 resonance with Jupiter. Also the clones that exhibit typical future evolution described in point 5 spent hundreds of years in the individual resonances with Jupiter (see evolution of clone \#26 in Fig. 2).

10. Statistically, the past orbital inclinations are smaller than future inclinations. The mean value of $i$ is about $12^{\circ}$ throughout the past evolution of 100 orbital clones, whereas it reaches about $17^{\circ}$ for future evolution (right-hand side of Fig. 5).
To ascertain whether the NG effects change the statistical conclusions, we repeated the numerical integrations for the case of a strictly gravitational approach. The two nominal orbits were taken from Model GRb (Table 5), and the next two sets of 100 clones of these nominal orbits were constructed; notice that orbit for past evolution was determined from the observations within another time period than orbit for the future evolution. Once again, each of cloned orbits was evolved backwards and forwards up to 8 thousand years. The resulting statistics were very similar to the one based on the NG evolution - the quantitative differences in the evolution of the orbital elements distribution are below the uncertainties resulting from the statistical approach.

\section{Comparison with $67 \mathrm{P} /$ Churyumov-Gerasimenko and 16P/Brooks 2}

The discovery of $81 \mathrm{P} /$ Wild 2 has a history that is analogous to the discovery of two other short-period comets: 16P/Brooks 2 and $67 \mathrm{P} /$ Churyumov-Gerasimenko, the future target of the Rosetta mission. The latter comet approached Jupiter to within 0.052 AU in February 1959, which reduced a perihelion distance from 2.74 AU to 1.28 AU. As a consequence, the comet was discovered in its second return to perihelion after the encounter with the giant planet. The change of orbit, however, was not as spectacular as in the case of $81 \mathrm{P} /$ Wild 2 . The past nominal orbit of 67P belonging to JS class (Horner et al. nomenclature) has transformed by the several moderate close encounters with Jupiter to the present orbit typical for JF comets (Królikowska 2003; Figs. 6 and 4 therein). There are also important differences in the past evolution of eccentricity and inclination for these two comets. The values of eccentricities in the past evolution of the 20 clones of nominal orbits of 67P were always below 0.6, whereas the values of inclinations were typically about $20^{\circ}-30^{\circ}$. Thus, from a dynamical point of view, the comet $81 \mathrm{P}$ seems a fresher object than comet 67P.

It is also interesting to compare the dynamical history of Wild 2 with the past evolution of $16 \mathrm{P} /$ Brooks 2 . The similarity in the orbital history of these comets was pointed out by Sekanina \& Yeomans (1985). Comet Brooks 2 was discovered by Barnard in August 1889 as a spectacular multiple object. It turned out that three years before its discovery, the comet passed only $0.001 \mathrm{AU}$ from Jupiter between the planet's ring and the orbits of Metis and Adrastea. This extremely close encounter with the giant planet split the cometary nucleus into multiple pieces. After that event, the main piece was observed as comet Brooks 2, and there has been no trace of the other fragments in later returns. The scenario of the nucleus splitting and the uncertainties in determination of the orbit of Brooks 2 were widely discussed by Sekanina \& Yeomans (1985). Here, though, we decided that the most appropriate starting orbit for the past evolution of the body that transformed into Brooks 2 is an orbit determined as a strictly gravitational fit to observations covering 7 years of two first apparitions (1889-1896). The dynamical integrations were performed 4000 thousand years back in time. The orbital evolution of the nominal orbit and its 20 clones is presented in Fig. 7. One can see that the 
evolution of the Brooks 2 orbit, with dramatic perihelion decreasing from 5.46 $\mathrm{AU}$ to 1.95 $\mathrm{AU}$ (aphelion: from 14.3 $\mathrm{AU}$ to $5.42 \mathrm{AU}$ ) due to the close encounter with Jupiter in July 1886, seems very similar to the evolution of Wild 2 . A more detail statistical analysis displays some differences mainly in the evolution of orbital eccentricity: Brooks 2 evolved backwards to statistically smaller $e$ in comparison to Wild 2. After 4000 thousand yr back, the mean $e$ determined from the evolution of 20 clones is about 0.48 , whereas for Wild 2 the mean $e$ reaches a value of 0.58 .

\section{Summary}

In this work, the symmetric and asymmetric models of NG effects in the 81P/Wild 2 motion were considered. Both types of model show moderate variations in the NG parameters, depending on which set of successive apparitions is used (see Tables 2 and 3). Nevertheless, the orbital linkage of all comet apparitions based on the constant parameters $A_{1}, A_{2}, A_{3}$ gives a satisfactory residual of 1'.27 (see Model Ia in Table 4). This symmetric model was used to show and then discuss the dynamical evolution of 100 orbital clones of the actual motion of $81 \mathrm{P} /$ Wild 2 within the time interval $\pm 8000 \mathrm{yr}$. In the most probable scenario of Wild 2 history a few thousands years ago, this comet was an object with its perihelion close to the Jupiter orbit and an aphelion distance greater than the Neptune orbit or even larger. From three short-period comets that experienced the very close encounter with Jupiter in the past (16P/Brooks, 67P/Churyumov-Gerasimenko, and 81P), Comet Wild 2 seems to be the freshest one in the inner solar system. Due to the extremely close approach to Jupiter in 1974, its future evolution differs substantially from its past evolution; and, with high probability, comet Wild 2 will survive as a typical Jupiter-family comet during the few thousands years that follow. After $8000 \mathrm{yr}$, however, the chance that the comet Wild 2 will still be a typical JF comet (with $q<3 \mathrm{AU}$ ) drops to $29 \%$.
In the second paper of these series we plan to model the NG perturbations of Wild 2 by introducing the discrete emission sources on rotating comet's nucleus. Then the positional observations as well as the observed water production curves will be used. Thus, in a forthcoming paper we will focus on the physical properties of the comet and its activity pattern.

Acknowledgements. The authors are deeply indebted to Professor Grzegorz Sitarski for many valuable discussions. We also wish to thank the referee for his constructive comments. This work was partly supported by the Polish Committee for Scientific Research (the KBN grant 4 T12E 039 28).

\section{References}

Bielicki, M., \& Sitarski, G. 1991, Acta Astron., 41, 309

Brownlee, D. E., Horz, F., Newburn, R. L., et al. 2004, Science, 304, 1764

Chesley, S. R., \& Yeomans, D. K. 2004, Proc. IAU Coll., 197: Dynamics of Populations of Planetary Systems

Farnham, T. L., \& Schleicher, D. G. 2005, Icarus, 173, 533

Horner, J., Evans, N. W., Bailey, M. E., \& Asher, D. J. 2003, MNRAS, 343,1057

Królikowska, M. 2003, Acta Astron., 53, 195

Marsden, B. G., \& Sekanina, Z. 1973, AJ, 78, 1118

Marsden, B. G., Sekanina, Z., \& Yeomans, D. K. 1973, AJ, 78, 211

Sekanina, Z. 2003, JGR 108, SRD 2

Sekanina, Z., \& Yeomans, D. K. 1985, AJ, 85, 2335

Sekanina, Z., Brownlee, D. E., Economou, T. E., Tuzzolino, A. J., \& Green, S. F. 2004, Science, 304, 1769

Sitarski, G. 1989, Acta Astron., 39, 345

Sitarski, G. 1994a, Acta Astron., 44, 91

Sitarski, G. 1994b, Acta Astron., 44, 417

Sitarski, G. 1996, Acta Astron., 46, 29

Sitarski, G. 1998, Acta Astron., 48, 547

Sitarski, G. 2002, Acta Astron., 52, 471

Weaver, H. A. 2004, Science, 304, 1760

Yeomans, D. K., \& Chodas, P. W. 1989, AJ, 98, 1083 